\title{
El principio de equidad en la Ley General del Ambiente: ética y justicia ambiental
}

\author{
IVÁN K. LANEGRA QUISPE*
}

SUMARIO: I. EL DERECHO AMBIENTAL DESDE LA ÉTICA: LA JUSTICIA AM-

BIENTAL.- II. JUSTICIA AMBIENTAL Y POLÍTICAS PÚBLICAS.- III. ¿CÓMO CONSTRUIR UN DERECHO AMBIENTAL JUSTO?.- IV. REFLEXIONES FINALES.

La relación entre la ética y el Derecho ambiental puede ser analizada desde distintas perspectivas. Hemos escogido el artículo X del Título Preliminar de la Ley General del Ambiente, ${ }^{1}$ que desarrolla el denominado «principio de equidad».

Según dicho principio, «el diseño y la aplicación de las políticas públicas ambientales deben contribuir a erradicar la pobreza y reducir las inequidades sociales y económicas existentes [...]». También de contribuir «[...] al desarrollo económico sostenible de las poblaciones menos favorecidas». Dentro del marco anterior, «el Estado podrá adoptar, entre otras, políticas o programas de acción afirmativas, entendidas como el conjunto coherente de medidas de carácter temporal dirigidas a corregir la situación de los miembros del grupo al que están destinadas, en un aspecto o varios de su vida social o económica, a fin de alcanzar la equidad efectiva».2

Nos proponemos, en primer lugar, justificar el interés del legislador en los problemas de equidad, que denominaremos «justicia ambiental». Luego, mostraremos algunos problemas que pueden derivarse de la aplicación del mencionado principio a partir del desarrollo de políticas públicas concretas. Finalmente, propondremos algunos mecanismos que pueden desarrollarse con el objetivo de incorporar la dimensión ética dentro del desarrollo del Derecho Ambiental en el Perú.

\section{EL DERECHO AMBIENTAL DESDE LA ÉTICA: LA JUSTICIA AMBIENTAL}

Como recientemente ha señalado Miguel Giusti, «la ética se refiere a esa experiencia de la mesura en la convivencia humana y a la conciencia de los

* Lima, 1971. Abogado por la Pontificia Universidad Católica del Perú y egresado de la Maestría en Ciencia Política de la misma universidad. Egresado del Programa de Derecho Ambiental Internacional y Comparado dellnstitutodelas NacionesUnidas paralaCapacitación ylalnvestigación-UNITAR.Trabajóen las unidades ambientales de los Ministerios de Salud, Transportes, Comunicaciones, Vivienda y Construcción, asícomo en el Consejo Nacional del Ambiente. Fue Gerente de Recursos Naturales y Gestión del Medio Ambiente del GobiernoRegionaldeJunín.ActualmentesedesempeñacomoAdjuntodelMedioAmbiente,ServiciosPúblicos y Pueblos Indígenas de la Defensoría del Pueblo. Es profesor de Elementos de Ciencia Política en la PUCP y docentedesu Diploma deEspecializaciónen DerechoAmbientaly delos Recursos Naturales. Lasopiniones delpresenteartículoserealizanatítulopersonalynocomprometenaningunadelasentidadesmencionadas.

1 Ley 28611.

2 Artículo X del Título Preliminar de la ley 28611. 
límites que no debieran sobrepasarse para hacerla posible». Como el mismo autor reconoce, ni dicho límite ni la conciencia han permanecido invariables a lo largo de la historia, aun cuando siempre hemos tenido la convicción sobre la necesidad de ambos. ${ }^{3}$ La aparición de la cuestión ambiental agrega a la conciencia y discusión sobre dichos límites una nueva dimensión.

Existen dos grandes paradigmas de la ética. Para el primero, denominado del bien común o de la felicidad, se trata de adecuar la conducta personal y social al sistema de valores de la comunidad donde uno vive. De esta manera, el problema ético es la identificación de dicho sistema de valores y virtudes que se expresa en la vida social. De otro lado, existe el paradigma de la autonomía o de la justicia, para el cual una sociedad justa para todos los seres humanos es una en donde todos los individuos, cualquiera fuera su sistema de valores personal, puede ejercer su libertad sin perjudicar a los demás. En este caso se trata ya no de buscar el sistema de valores a seguir, sino la regla general de imparcialidad que haga posible la coexistencia de concepciones valorativas rivales entre sí. Aunque la necesidad de construir un sistema de valores compartido sobre la relación entre el ser humano y el medio que lo rodea es una tarea también importante, quisiera ahora centrarme en el segundo paradigma. Es decir, deseamos preguntarnos sobre cómo, en un país pluricultural y complejo como el nuestro, podemos construir reglas justas que rijan las acciones y relaciones humanas que tienen un impacto relevante sobre el ambiente. ${ }^{5}$

El punto de partida para dar respuesta a dicha pregunta pasa por determinar las condiciones ambientales deseadas por todos, es decir, definir el contenido del derecho a un ambiente adecuado. A partir de este primer paso requerimos identificar las acciones y relaciones humanas que pudieran generar riesgos sobre dichas condiciones, para luego definir los mejores medios para enfrentar dichos riesgos. Estas operaciones son traducidas a instituciones jurídicas, y derivan en un sistema de asignación legal de los bienes ambientales, así como de los costos de las cargas y riesgos medioambientales entre los distintos actores de la sociedad; ello genera a su vez obligaciones y responsabilidades específicas, cuya concreción, sin embargo, no depende totalmente de la legislación ambiental, sino de la institucionalidad política y jurídica como un todo. La finalidad última del sistema jurídico, en su dimensión ambiental, es la de asegurar el mantenimiento de las condiciones ambientales mínimas o, dicho en términos jurídicos, garantizar la posibilidad efectiva del goce del derecho a un ambiente adecuado donde vivir.

3 Gıustı, Miguel. El soñado bien, el mal presente. Rumores de la ética. Lima: Fondo Editorial PUCP, 2008, p. 18.

4 lbid., pp. 32-48.

5 Yahemos ensayado una definición de Derecho Ambiental. Lo entendimos como aquella área del Derecho que «estudialaregulaciónjurídicadelasaccionesyrelacioneshumanasqueimpactansobrelascondiciones ambientales que en conjunto son consideradas necesarias para la buena vida de la sociedad y para la proteccióndelaspersonas, asícomoparael buenfuncionamientodelosecosistemas". LANEGRA QUISPE, Iván Kriss. El derecho ambiental: conceptos y tareas. Thémis. Revista de Derecho, no 56, agosto de 2008, Lima, p. 10. 
Esta perspectiva implica que el Derecho Ambiental debe resolver problemas de justicia. ${ }^{6}$ Esto refiere a criterios éticos y políticos para asignar las cargas y riesgos, proceso que alcanza mayor intensidad al definir tanto el contenido del derecho a un ambiente adecuado, como al plantear los objetivos de la política ambiental.

Los problemas de justicia son también centrales cuando uno debe decidir, en un contexto de restricciones, qué problemas debieran ser atendidos primero. Como es natural, deberíamos enfocarnos en aquellos que afectan lo que valoramos más, por ejemplo, los riesgos ambientales que reducen significativamente la expectativa de vida o que causan más muertes en comparación con otros factores. Y todo esto sin perder de vista la cuestión de la justicia ambiental intergeneracional. No se trata solo de pensar en las consecuencias para las generaciones actuales: también se debe considerar, con todas las dificultades de pensar y decidir sobre un futuro desconocido, a las generaciones aún por venir.?

Es aquí donde alcanza todo su valor el principio de equidad. Esta palabra proviene del latín aequitas, de aequus, 'igual', y alude a la justicia natural, en oposición a la «ley positiva». ${ }^{8}$ Por lo tanto, se alude como norte de la acción social en materia ambiental la reducción de las desigualdades que se consideran injustificadas, en materia socioeconómica. ¿Cómo incide la política ambiental en la inequidad?

\section{JUSTICIA AMBIENTAL Y POLITTICAS PÚBLICAS}

Una de las características centrales del ambiente, desde el punto económico, es su carácter de bien público. Es decir, cuando se provee un ambiente de buena calidad, este es disfrutado por todos sin excepción, y todos también sufrimos, en distintos grados, los males de su deterioro. Del mismo modo, una vez puesto a disposición, el costo por incorporar a una persona más al goce del bien suele ser irrelevante. ${ }^{9}$ No obstante, la pérdida de calidad ambiental implica un costo que no todos enfrentan en igualdad de condiciones. Una persona pobre enfrenta

6 Porjusticia(social)entendemoselmodoenelcuallosbienes(ventajas)ymales(cargas)deberíanserdistribuidosentrelos miembros de una sociedad humana. (MILLER, David. Principles of SocialJustice. Londres: Harvard University Press, 2003, p. 1). En este marco, es el modelo de la justicia distributiva el más comprensivo. Sin embargo,algunosautoresproponenirmásalláyrevisarlosmodelostradicionales dejusticiaparaincorporar lasespecificidadesdelo «ecológico»o «ambiental».VICENTEJıMÉNEZ,Teresa. «Elnuevoparadigmadelajusticia ecológica». En Justicia ecológica y protección del medio ambiente. Madrid: Trotta, 2002, pp. 59-67.

7 Alrespecto,elprincipiodesostenibilidadrecogidoenelartículoV delTítuloPreliminardelaLeyGeneraldel Ambiente establece que la gestión ambiental y el ejercicio y la protección de los derechos ambientales «se sustentan en la integración equilibrada de los aspectossociales, ambientales yeconómicos deldesarrollo nacional, así como en la satisfacción de las necesidades de las actuales y futuras generaciones". Frentea este problema, Teresa Vicentenos plantea, citandoa Visser't Hooft, que los seres humanos pertenecemos a una "empresa colectiva de continuación» de la especie humana, frente a la cual el mantenimiento de nuestraestructurabásicadesupervivenciaobligaanoconsiderarsololosinteresesindividualesycolectivos existentes, sinotambiénlacontinuidad,expresadaenlasfuturasgeneraciones, demantenerdichaestructura básica. VICENTE JIMÉNEZ, Teresa. «Orden ambiental - orden jurídico, interdependencia, participación y condicionalidad". En Justicia ecológica y protección del medio ambiente. Op. cit., pp. 53-54. Nos encontramos frente a un interés supraindividual y supracolectivo, que comprende a la especie humana entera.

8 Vigésima segunda edición del Diccionario de la Lengua Española de la Real Academia Española.

9 Laexcepción pasa por la capacidad de carga de bienes ambientales, que pueden ser disfrutados solo bajo condicionesrestrictivasdeacceso: porejemplo,gozardelabellezadelParqueNacionaldelManucuandolas visitas están limitadas estrictamente a lo sostenible. 
mayores restricciones frente a la contaminación ambiental o a la pérdida de ecosistemas o de servicios ambientales. Por dicha razón, los gobiernos tienen un papel crucial en la conservación y el aprovechamiento sostenible del capital natural como herramienta en la lucha contra la pobreza y la desigualdad. Invertir en el capital natural supone protegerse contra riegos naturales, controlar los vectores de las enfermedades y las plagas, y preservar los servicios que presta el ecosistema para mantener la productividad de las cosechas y evitar los vertidos tóxicos en el agua y en el aire. ${ }^{10}$

De otro lado, la pobreza también puede tener un impacto sobre el ambiente. Con frecuencia las comunidades pobres de las zonas rurales utilizan los recursos naturales en formas que, aunque alivian transitoriamente las necesidades insatisfechas, terminan por derivar en pérdidas permanentes de capital natural. La ocupación de áreas frágiles o de gran biodiversidad, incapaces de sostener actividades agrícolas tradicionales, o los asentamientos humanos permanentes, están impulsados en varios casos por la situación de pobreza de la población. Otros ejemplos son el uso de leña como combustible y la depredación de fauna silvestre para la alimentación o la venta comercial, en muchas ocasiones ilegal, por parte de comunidades pobres con objetivos de simple subsistencia. Al mismo tiempo, otros actores pueden obtener importantes beneficios de dichas actividades ilegales. Otras dimensiones en las que se cruzan los temas de sostenibilidad y equidad, no menos importantes, están asociadas con la ocupación, por parte de poblaciones campesinas, de ecosistemas agrícolas muy deteriorados o con la concentración de problemas ambientales severos en algunas comunidades pobres en las ciudades, entre las que se incluyen la falta o mala calidad de servicios de saneamiento básico, el aire altamente contaminado y los suelos inestables o muy vulnerables en barrios pobres. ${ }^{11}$

No tenemos muchos estudios detallados sobre la relación entre la pobreza y el ambiente. No obstante ello, la epidemia de cólera que azotó el país en 1991 y los problemas ambientales originados por la expansión del narcotráfico parecen ser buenos ejemplos de los vínculos entre estas dos variables. ${ }^{12}$ De otro lado, debemos comprender que el desarrollo no puede comprenderse solo con un tema de ingresos económicos o de provisión de bienes:

[...] en la definición de desarrollo ya no se trata solamente de cuántas cosas se producen por las personas (productividad), ni de cómo se las reparten entre ellas (equidad), ni de si dichas cosas alcanzan para vivir o desempeñarse adecuadamente en la vida (necesidades básicas). Se trata más bien de saber cuántas vidas y cuánta vida, entendidas como capacidades y desempeños, se logran con la utilización de ciertas cosas por el ser humano. ${ }^{13}$

10 SACHS, Jeffrey. El fin de la pobreza. Cómo conseguirlo en nuestro tiempo. Bogotá: Debate, 2006, pp. 355-356 y 360.

11 Ocampo, José Antonio. Políticas e instituciones para el desarrollo sostenible en América Latina. Santiago de Chile: CEPAL, 1999, p.11.

12 Gonzales de Olarte, Efraín. Medio ambiente y pobreza en el Perú. Lima: IEP, 1997, p. 98.

13 Iguiñız, Javier. Definiciones de desarrollo y experiencias de género. Lima: Instituto Bartolomé de Las Casas, 1996, p. 8 
Debemos a Amartya Sen la concepción del desarrollo «como un proceso de expansión de las libertades reales de que disfrutan los individuos». Esto es lo que denomina la «visión del desarrollo como libertad», la cual cambia la perspectiva aún dominante en el tema y centra la atención en los fines antes que en los medios, por lo cual esta visión «exige la eliminación de las principales fuentes de privación de libertad», lo que incluye la pobreza y la tiranía, la escasez de oportunidades económicas, las privaciones sociales sistemáticas, así como la intolerancia o el exceso de intervención de los Estados represivos. Cuando se tiene el enfoque amplio de "concebir el desarrollo como un proceso integrado de expansión de libertades fundamentales relacionadas entre sí», es posible «apreciar simultáneamente el vital papel que desempeñan en el proceso muchas y diferentes instituciones». Entre estas últimas, podemos encontrar tanto a los mercados como a los gobiernos -en todos sus niveles-, los partidos políticos y otras instituciones ciudadanas, los sistemas educativos «y las oportunidades de diálogo y debate públicos». Además, este «enfoque nos permite reconocer el papel de los valores sociales y de las costumbres vigentes, que pueden influir en las libertades de que disfrutan los individuos y que tienen razones para valorar». Estas normas compartidas pueden influir, entre otros elementos, en el tratamiento del ambiente. ${ }^{14}$

Múltiples estudios demuestran que la contaminación ambiental es la causa de innumerables enfermedades, que afectan directamente la calidad de vida de la gente. ${ }^{15}$ Entre ellos, los niños y las personas con menos recursos constituyen los grupos más vulnerables. Se calcula que, en niños de entre 0 y 14 años de edad, el porcentaje de muertes que podían atribuirse al medio ambiente equivale a un 36\%. Mientras el 25\% de todas las muertes registradas eran atribuibles a causas ambientales en las regiones en desarrollo, en las desarrolladas el porcentaje solo llegaba al $17 \% .{ }^{16}$ Las políticas ambientales, desde esta perspectiva, en tanto contribuyen a reducir las amenazas sobre las personas — sobre su salud y su bienestar en general—-, aportan al proceso de expansión de libertades y están plenamente integradas a otras políticas. Por lo tanto, privar a una persona de un ambiente saludable es un ataque contra su dignidad.

Los gobiernos de los países en desarrollo han aplicado políticas destinadas a mejorar los ingresos económicos de sus habitantes, por lo que han ampliado la cobertura de servicios y el acceso a bienes básicos. No obstante, y he aquí el elemento más polémico, en ocasiones en el intento por asegurar el goce de ciertas libertades ponemos en riesgo otras. ¿Qué

14 SEn, Amartya. Desarrollo y libertad. Bogotá: Planeta, 2001, pp. 19-20, 25 y 26.

15 «Secalculaqueel $24 \%$ delacargademorbilidadmundialyel $23 \%$ detodoslosfallecimientospuedenatribuirse a factores ambientales. De las 102 principales enfermedades, grupos de enfermedades y traumatismos que cubre el Informe sobre la salud en el mundo de 2004, los factores de riesgo ambientales contribuyeron a la cargademorbilidaden 85categorías. Lafraccióndelamorbilidadatribuibleespecíficamentealmedioambiente variaba de manera notable entre las diferentes enfermedades. Se calcula que en todo el mundo el $24 \%$ de la cargademorbilidad(añosdevidasanaperdidos)yaproximadamenteel23\%detodaslasdefunciones(mortalidad prematura)eran atribuibles afactoresambientales». Prüss-Üstün, Ay C. Corvalán. Ambientessaludables y prevención de enfermedades: hacia una estimación de la carga de morbilidad atribuible al medio ambiente: resumen de orientación. Ginebra: Organización Mundial de la Salud, 2006, p. 5.

EL PRINCIPIO DE EQUIDAD EN LA LEY GENERAL DEL AMBIENTE: ÉTICA Y JUSTICIA AMBIENTAL 
hacer frente a ello? ¿Cómo garantizar el acceso a la salud, a la educación, a un trabajo digno, sin poner en riesgo el derecho a un ambiente saludable? Recientemente, el Perú ha promovido fuertemente las inversiones, en especial del aprovechamiento de nuestros recursos naturales no renovables. Buscamos obtener los recursos que nos sirvan para satisfacer el primer grupo de derechos. Pero, al inicio, esta política careció de políticas públicas ambientales que evitasen poner en riesgo el derecho a un ambiente adecuado. Aunque ahora existe un mayor desarrollo, queda un buen trecho por recorrer. Además, pareciera que ciertos países, como el Perú, tendrían que enfrentar un problema adicional: la estructura de incentivos que genera la presencia de una economía sustentada fundamentalmente en el aprovechamiento de los recursos naturales tiende a agudizar los efectos de la inequidad.

En un país sin instituciones fuertes, se genera una competencia por el control de los recursos naturales, basada no en el bien público, sino en el poder socioeconómico de los actores y sus objetivos privados. Esto lleva normalmente a una situación de inestabilidad política y corrupción, y promoverá la aparición de gobernantes autoritarios que se aprovecharán del propio Estado para controlar los recursos con fines particulares. Este proceso, mediado simplemente por la acumulación de poder, llevará a la concentración de la riqueza en pocas manos, la de los actores más fuertes, lo cual generará situaciones de gran inequidad. Esto afecta la cohesión social y socava la fe en la economía de mercado, pues la riqueza no se adquiere por el trabajo duro, que legitima su obtención, sino por la simple apropiación, alcanzada en ocasiones por medios ilegítimos o ilegales. El descontento de los «perdedores» en estas luchas, que son la gran mayoría, constituye el combustible de conflictos sociales. ${ }^{17}$

\section{III. ¿CÓMO CONSTRUIR UN DERECHO AMBIENTAL JUSTO?}

Si debemos adecuar nuestras instituciones y organizaciones al problema de la gestión y distribución de los riesgos, la pregunta que surge es: iqué mecanismos utilizar para poder llegar a acuerdos en materia de justicia ambiental? Es decir, icómo definir la distribución de los beneficios ambientales y sus riesgos en el interior de nuestra sociedad, sin caer en los riesgos de la imposición de intereses privados, como si fueran públicos, o de la dictadura de los expertos, que excluye a los ciudadanos?

El diseño y la implementación de las políticas ambientales han sido percibidos como procesos esencialmente técnicos, por lo cual han involucrado un número limitado de actores, a los que se denomina «expertos», en espacios inaccesibles al público. ${ }^{18}$ Detrás de esta posición se encuentra la pretensión de que es posible hallar soluciones técnicas 
políticamente neutrales. Pero, en América Latina, no se ha considerado con seriedad si tiene asidero dicha pretensión; es decir, si es posible generar una política ambiental científicamente objetiva y neutra, y si se cuenta en todos los casos con el conocimiento científico-técnico necesario para tomar decisiones más allá de las opiniones de los grupos de interés. Es obvio que nos encontramos muy lejos de aquella situación. ${ }^{19} \mathrm{Y}$ aun en el caso de contar con la información necesaria, la visión técnica tiende a reducir a un solo factor la valoración de las distintas opciones que se presentan en el momento de elaborar una política ambiental, o al tomar una decisión que involucre factores ambientales. ${ }^{20}$

Si las evaluaciones que realizan los actores están separadas por distintas valoraciones, e inclusive por distintas perspectivas culturales, entonces la decisión o política finalmente elegida tendrá que, o reflejar la posición de uno de los grupos, o llevar las cosas a una suerte de transacción imperfecta, que podría cuestionar severamente la legitimidad del proceso. La consagración de unos valores por la simple exclusión de otros trae consigo la imposición de una cosmovisión del mundo, o por lo menos la imposición de intereses particulares sin el reconocimiento de los intereses opuestos y sin posibilidad de la construcción de un interés común.

Esta situación puede derivar en situaciones de marginalidad y de violencia. No es más auspiciosa una transacción imperfecta, en el sentido de llegar a un acuerdo como si se tratara de un asunto de suma-cero - lo que yo gano tú lo pierdes-, una suerte de regateo sobre intereses en pugna. El tema es que para realizar dicha transacción se requiere igualmente traducir los intereses a una sola escala de valores, la cual sirva de referencia para la transacción. Además, no siempre es posible realizar compensaciones —es decir, dar a algo a cambio de otra cosa—, pues en muchas ocasiones el interés principal en pugna no es compensable, y cualquier intento por forzar dicha transacción significaría, en términos prácticos, producir resultados en los cuales será fácil leer el triunfo de los intereses de solo una de las partes.

Incluso, moviéndonos en un plano estrictamente científico, empieza a generarse un consenso sobre el hecho de «que los ecosistemas no solo son más complejos de lo que se pensaba, sino además más complejos de lo que podemos pensar, estableciéndose así un límite cognitivo a nuestra comprensión científica». ${ }^{21}$ Esto debería bastar para demostrar las claras limitaciones de pensar la definición de las políticas ambientales como un proceso exclusivamente técnico-científico.

Con todo lo anterior no se quiere negar el papel importante que juegan la tecnología y la ciencia en la mejora de la calidad de vida

19 GudynAs, Eduardo. «Incertidumbre, riesgo y conocimiento experto: implicaciones para las políticas ambientales". En Rodrigo Araya Dujisin. Ecología de la información. Escenarios yactores para la participación ciudadana en asuntos ambientales. Caracas: Editorial Nueva Sociedad, 2001, pp. 17-32 y 20.

20 Martínez Alier, Joan y Jordi Roca Jusmet. Economía ecológica y política ambiental. Ciudad de México: FCE, 2000, pp. 257-269.

21 Gudynas, Eduardo. Op. cit., p. 21. 
de la población, en la toma de mejores decisiones y en su valiosa contribución en la búsqueda de nuevas formas de evitar el deterioro ambiental; tampoco se niega la importancia de las decisiones individuales sobre cualquier política ambiental. Finalmente, el impacto ambiental es resultado de la suma de millones de decisiones individuales agregadas. Sin embargo, incluso la ciencia moderna tiene límites. Detrás de los sistemas que determinan desde la economía o la ciencia cómo y cuánto se debe producir y consumir, se esconde una agenda política inevitable que tiene que ser encarada. La legitimidad de las políticas ambientales, pues, no puede sostenerse solo de la ciencia o la técnica. ${ }^{22}$ Dado que es imposible alinear todos los riesgos a categorías únicas - la valoración económica-, entonces se requiere emprender algún tipo de proceso alternativo. Ese proceso es, desde nuestro punto de vista, político. Y lo es en dos sentidos. Por un lado, implica la gestión de conflictos sociales y no la simple aplicación de métodos de análisis técnico con pretensiones de objetividad y neutralidad científica. En consecuencia, como criterio para diseñar políticas públicas ambientales, no basta una racionalidad instrumental o estratégica. Es indispensable, de acuerdo con Jürgen Habermas, una racionalidad comunicativa, es decir, introducir una teoría de la justicia sostenida en el discurso y el diálogo. Los fines no están preestablecidos y requieren discutirse.

Pero, el proceso para enfrentar los riesgos, es político en otro sentido. Hace necesario estructurar políticamente a la sociedad a través de temas como lo ambiental, con lo cual se rescata su naturaleza pública. La incertidumbre y el potencial caos generan tensión sobre la sociedad, lo que no es sencillo de manejar. En muchas ocasiones, se suele caer en la tentación de reducir la incertidumbre mediante la búsqueda de una respuesta definitiva y unificadora de las evaluaciones culturales y éticas; un acto de puro poder que devuelva la distinción entre lo bueno y lo malo, y que elimine, como sugiere Ulrich Beck, el carácter de distorsión del eso y aquello. Se trata de volver a la seguridad del o eso o aquello. Es dejar en manos de unos cuantos, o de simplemente uno, la decisión definitiva. Los fundamentos que puede utilizar quien decide son la técnica, la ciencia, la tradición o incluso la religión. En contraposición, podemos apostar por no negar la ambivalencia del eso y aquello. Esto implica una construcción política que se adapte a las nuevas condiciones. Abrir un espacio de encuentro de perspectivas distintas sin recurrir a las exclusiones solo puede llevar a una apuesta por la democracia. De lo que se trata es de alcanzar un equilibrio entre el papel de los expertos $-\mathrm{y}$ del conocimiento experto - y los mecanismos democráticos, y mantener al mismo tiempo bajo control la influencia de grupos sectoriales organizados. La política ambiental es un territorio fértil para la discusión teórica y las respuestas institucionales y organizacionales frente a este desafío. 
De acuerdo con Claudia Feres, la teoría democrática dominante sostiene que el poder político

[...] puede organizarse democráticamente a través de instituciones que median la relación entre los intereses privados de los individuos y el propio poder. La legitimidad del gobierno residiría en la voluntad de los individuos organizada a través del principio de la mayoría, dado que la posibilidad de la unanimidad parece ser un ideal contrafactual [sic] en las sociedades modernas. Decisiones legítimas, por tanto, se construyen cuando se calcan de la voluntad de la mayoría y no de la de todos.

De dicha forma, se obtendría, además, eficiencia en el proceso de toma de decisiones. ${ }^{23}$ Corresponde a la población elegir a los gobernantes mediante elecciones periódicas, mientras que corresponde al gobierno tomar decisiones basadas sobre criterios de racionalidad técnica. Así, sería facultad exclusiva del Estado definir lo más apropiado en materia ambiental mediante conocimientos de expertos en el interior de sus procesos técnicos. Dentro de esta lógica, cualquier intento de participación por fuera de ellos podría implicar una amenaza contra la racionalidad de las decisiones.

Pero ies posible pensar en otra base de legitimidad? ¿Tiene la noción de decisiones colectivas sobre el ejercicio del poder algún asidero en las sociedades complejas? La contribución de Jürgen Habermas y de muchos de los autores que han aprovechado sus elaboraciones teóricas - Cohen y Arato, Cunill Grau, Avritzer, Feres, Bohman, Cohen, etcéteraapunta a una respuesta afirmativa. La propuesta, conocida como teoría democrática deliberativa, «afirma que el proceso de decisión del gobierno tiene que sustentarse en la deliberación de los individuos racionales en foros amplios de debate y negociación. Esa deliberación no resulta de un proceso agregativo de las preferencias fijas e individuales sino de un proceso de comunicación, en espacios públicos, que antecede y auxilia la propia formación de la voluntad de los ciudadanos». ${ }^{24}$

Habermas ha construido una teoría que permite concebir un espacio, la esfera pública, en donde es posible llevar adelante este proceso de discusión pública y de argumentación, más allá de los mecanismos de toma de decisiones derivados de la regla de la mayoría. Es un espacio donde los individuos interactúan unos con otros; debaten las decisiones tomadas por la autoridad política, así como los contenidos morales de las relaciones existentes en el nivel de la autoridad social, y presentan demandas con relación al Estado. ${ }^{25}$

EL PRINCIPIO DE EQUIDAD EN LA LEY GENERAL DEL AMBIENTE: ÉTICA Y JUSTICIA AMBIENTAL 
Aquí debemos mencionar el concepto de sociedad civil, ${ }^{26}$ término de uso muy difundido, a pesar de la imprecisión con que se utiliza ¿Qué características debería tener un espacio societal para ser considerado como sociedad civil? Para Cohen y Arato, ésta, en tanto modelo normativo distinto del Estado y del mercado, requiere pluralidad — muchos tipos de asociaciones-, publicidad -instituciones culturales y de comunicación - , privacidad — un espacio para la reflexión individual- y legalidad - un marco normativo que precise las otras condiciones-. Una sociedad civil vital requiere de una cultura política acostumbrada al ejercicio de la libertad: necesita un mundo de la vida ya racionalizado - es decir, comunicativo- Si no, pueden aparecer movimientos populistas ciegamente tradicionales - tradiciones endurecidas y anquilosadas-, que pueden ser modernos en sus objetivos pero antidemocráticos en sus formas - no racionales, no comunicativos-. Esto es especialmente relevante dentro de una realidad como la peruana, en la que grupos de esta naturaleza intervienen en la discusión pública de lo ambiental. En segundo lugar, solo cuando la opinión pública supera los filtros del procedimiento institucionalizado, se convierte en poder comunicativo - $y$ no antes- Y Y tercero, la sociedad civil no reemplaza al Estado — ni busca hacerlo-, pero tampoco el poder administrativo puede producir sociedad civil, aunque sí puede generar condiciones para su aparición. ${ }^{27}$ Por las razones expuestas, la simple existencia de espacios de participación, o su apertura, no genera participación, ni menos discusión comunicativa. Tampoco conlleva una situación de sometimiento de la sociedad civil a las políticas públicas. Aquella, usando el lenguaje ordinario de ciudadanos legos, puede realizar sus propias traducciones de los lenguajes especiales de los subsistemas - como es el caso del lenguaje técnico especializado de las ingenierías y de las ciencias naturales en la discusión ambiental, o también de la economía y la gestión pública-, cosa que, por cierto, también hace el Estado —a través de sus burocracias o mediante la contratación de dichos especialistas-, el cual requiere servirse del resto de subsistemas para subsistir. No solo requiere del subsistema económico —-mediante los tributos—; también necesita del sociocultural, con sus productos simbólicos, y el manejo de la ciencia y la técnica como herramientas legitimadoras de accionar.

Por lo tanto, la gestión ambiental no requiere solo de información técnica precisa, también necesita de espacios democráticos de participación y de discusión pública sobre los fines ambientales y sobre las prioridades de la sociedad respecto de los distintos bienes públicos y de los propios

26 La sociedad civil de Jürgen Habermas no es la de Hegel o la de Marx. Este último concibió a la sociedad burguesa como el sector privado, el mercado. En contraste, la sociedad civil en Habermas se encuentra claramente diferenciada del mercado y del poder administrativo estatal, los cuales son concebidos como sistemas. Lasociedadciviles unatramaasociativa, quenoesnielEstadonielmercado, yquetieneunabase voluntariay una vocación pública.Anclalasestructurascomunicativas delespaciodelaopinión públicaen elmundodelavida,conlocualinstitucionalizalos discursosyorganizalosespaciosdediscusión. HABERMAS, Jürgen. Facticidad y validez. Sobre el derecho y el Estado democrático de derecho en términos de teoría del discurso. Madrid: Trotta, 1998, pp. 446-448.

27 lbid., pp. 395-396 y 495-508. 
intereses particulares; no debe huir de los conflictos, sino aprovecharlos en su potencial renovador de la política, dichos espacios no pueden permanecer aislados de la discusión general sobre los fines de la sociedad, ni pretender la desaparición de los conflictos. El ámbito natural de estos procesos ha sido el parlamento, pero esta institución se ha visto también sujeta por presiones de intereses particulares y sometida a incentivos que la llevan a priorizar la transacción de intereses por sobre la búsqueda del interés común.

Los intentos institucionales explorados hasta la fecha, sin embargo, no han mostrado mucho éxito en esta tarea. Por un lado, la participación en la Evaluación de Impacto Ambiental de Proyectos ha mostrado sus limitantes, en tanto espacio dominado por la lógica técnica; otros mecanismos como las Comisiones Ambientales (Regionales y Municipales) han resultado ser más exitosos en la definición de las metas ambientales, pero menos eficaces al vincular estas definiciones con decisiones efectivas de gobierno, y menos aun para vincular la agenda ambiental con la agenda del desarrollo. Esto no implica que estos espacios deberían abandonarse, sino que deberían ser mejorados para enfrentar los desafíos descritos. Por ejemplo, icómo vincular el desarrollo de agendas ambientales a escala local y regional con la definición de la agenda nacional?

$\mathrm{Al}$ respecto, pareciera que procesos de discusión vinculados con la planificación del territorio y la discusión directa de la variable ambiental dentro de procesos de toma de decisión de alto nivel presentan mejores perspectivas para la incorporación de una discusión pública y la inclusión de los criterios establecidos en el principio de equidad. Es el caso de la herramienta Zonificación Ecológica Económica, que sugiere integrar las necesidades del desarrollo a las capacidades naturales del territorio. Otro ejemplo es la Evaluación Ambiental Estratégica —de políticas, planes y programas-, que busca incorporar el componente medioambiental en el diseño de políticas públicas de otras áreas. El análisis, en ambos casos, debe considerar factores sociales y, concretamente, los problemas de inequidad y exclusión existentes. Finalmente, en el propio diseño de las políticas ambientales, pueden establecerse directrices o lineamientos para asegurar la consideración de los problemas de equidad. ¿No es acaso prioritario atender los problemas de contaminación que afectan la salud de una población que posee pocas posibilidades de enfrentar sus riesgos, incluida la salida del sitio contaminado, como en el caso de La Oroya? ¿No deberíamos considerar políticas que faciliten la participación de la población con menores recursos, pero que asuman los costos directos de nuevos proyectos?

Por último, el papel de actores como la prensa, los centros académicos y las organizaciones no gubernamentales resulta también fundamental, en tanto estas instituciones pueden contribuir precisamente a una discusión pública de la agenda ambiental y a su incorporación en la agenda del desarrollo. De hecho, los medios de comunicación han aumentado

EL PRINCIPIO DE EQUIDAD EN LA LEY GENERAL DEL AMBIENTE: ÉTICA Y JUSTICIA AMBIENTAL 
durante los últimos años la cobertura de la agenda ambiental, aunque básicamente con fines de cobertura noticiosa de conflictos y de ilustración de los problemas. Ejemplo reciente de lo primero es la cobertura de los conflictos mineros; de lo segundo, el tratamiento de un tema como el cambio climático y sus efectos.

\section{REFLEXIONES FINALES}

El principio de equidad plantea un desafío ante el diseño de las políticas ambientales. Lo obliga a mirar más allá de su ámbito, a integrarla con la agenda del desarrollo y de la lucha contra la pobreza. También hace urgente repensar los criterios legales y políticos necesarios para producir una justa distribución de las cargas y los riesgos ambientales entre los ciudadanos, así como para la protección de los grupos más vulnerables. Un contexto de restricción de recursos económicos torna aun más importante este tema. La mejora de la calidad ambiental amplía las posibilidades de desarrollo de las personas y aumenta sus libertades reales. Su deterioro, en cambio, afecta en mayor grado a los más vulnerables.

Las instituciones políticas no pueden declararse neutrales ante esta situación, y, conforme con el principio de equidad de la Ley General del Ambiente, deben asumir un sesgo positivo en favor de los menos favorecidos. Este es un criterio que incluso justifica el desarrollo de programas especialmente dirigidos a atender dicho objetivo. Sin embargo, sin mecanismos democráticos de control de este proceso, intereses privados pueden pasar por públicos, justificados por la técnica o la economía. Para evitar ello, se requiere una discusión democrática basada sobre razones. Aquí se enlaza el derecho a la participación ciudadana ambiental, también reconocido por la Ley General del Ambiente. Esto último no se logrará sin una apuesta ciudadana por construir una sociedad civil activa en materia ambiental. Para ello, es indispensable enlazar la agenda medioambiental con los derechos fundamentales, en especial con la salud, y con la satisfacción de las necesidades básicas. Este es el camino para construir una legislación y políticas públicas ambientales que cumplan con el principio de equidad.

Finalmente, algunas palabras sobre el papel de los abogados: incluir esta perspectiva de derechos y de equidad en las distintas tareas dentro del ejercicio profesional es una primera obligación. Sin esta dimensión, la práctica legal será solo un instrumento de procesos que profundizan los problemas de inequidad. En cambio, desde la mirada de una auténtica responsabilidad social, la cohesión social se convierte en un componente crucial para la sostenibilidad de los negocios y en un requisito para el desarrollo justo. Se alcanzaría así el fin último del Derecho: el bienestar de las personas y de las futuras generaciones. 